(C) 2016 IEEE. Personal use of this material is permitted. Permission from IEEE must be obtained for all other uses, in any current or future media, including reprinting/republishing this material for advertising or promotional purposes, creating new collective works, for resale or redistribution to servers or lists, or reuse of any copyrighted component of this work in other works. 


\section{Optimization of the Variable Speed Pump Storage Participation in Frequency Restoration Reserve Market}

\author{
Jorge M. Filipe \\ Carlos L. Moreira \\ INESC TEC and Faculty of Engineering, University of Porto \\ Porto, Portugal \\ \{jorge.m.filipe, carlos.moreira\}@inesctec.pt
}

\author{
Ricardo J. Bessa \\ Bernardo A. Silva \\ INESC TEC - INESC Technology and Science \\ Porto, Portugal \\ \{ricardo.j.bessa, bernardo.a.silva\}@inesctec.pt
}

\begin{abstract}
Several countries have a significant installed capacity of large-scale reversible hydro power plants. This large-scale storage technology comes with high investments costs, hence the constant search for methods to increase and diversify the sources of revenue. Traditional fixed speed pump storage units typically operate in the day-ahead market to perform price arbitrage and, in specific cases, provide downward replacement reserve (RR). Variable speed pump storage can not only participate in RR but also contribute to frequency restoration reserve (FRR), given their ability to control its operating point in pumping mode. This work proposes a strategy to manage the water resource and maximize the power plant revenue by participating in the dayahead market but also providing ancillary services. Moreover, a model to correctly allocate the water resource throughout the year is presented, as well as an evaluation module to calculate the real revenue of the system.
\end{abstract}

Index Terms-Pumped Storage Power, Day-ahead Market, Replacement Reserve, Frequency Restoration Reserve, Forecasting

\section{INTRODUCTION}

Reversible hydro power plants with reservoir (Pumped Storage Power - PSP) are the most mature storage technology with high integration levels in countries such as Portugal, Spain and Switzerland. Typically, the fixed speed PSP participate in the electricity market for price arbitrage (i.e., pump in low price periods and generate in high price periods) and provision of downward replacement reserve (RR) in specific cases. Due to technical limitations, the current fixed speed PSP technology does not participate in the frequency restoration reserve (FRR). In contrast, variable speed PSP units can not only participate in RR but also in FRR, given their flexibility to quickly change the consumption operating point.

With the increasing penetration of renewable energy sources in the generation mix and the consequent decrease in the

This work is being carried out as a part of the HYPERBOLE project (HYdropower plants PERformance and flexiBle Operation towards Lean integration of new renewable Energie) - hyperbole.epfl.ch. This project is funded by the European Commission under the seventh framework program (FP7) under grant agreement number 608532. global inertia of the system, several works have begin to study the performance and reliability of variable speed PSP in the electrical grid.

In [1] it is analyzed the technical benefits achieved by replacing the existing fixed speed pump units by variable speed ones in an Indian PSP unit, which would be the first time in that country. The conclusion is that the the benefits are significant since adds frequency regulation in a location where the nominal frequency is constantly below $50 \mathrm{~Hz}$. [2] and [3] explore the use of a ternary unit operated in hydraulic short circuit to provide active power control in pumping mode and concludes that it improves the Virtual Power Plant output power and thus the power network frequency stability in case of significant solar power variations. In [4] it is presented an overview of the trends and challenges in PSP, which shows that ancillary services markets, particularly those related to balancing supply and demand, emerge as a valuable source of revenue.

To our knowledge, in the literature only the work of [5] formulates an optimization problem for variable-speed PSP participation in the FRR market, which is then used to estimate the maximum theoretical revenue of the system. The work assumes perfect knowledge of market prices and water inflows and that the amount of energy to be used from the total reserve band available is know at the time of the optimization.

The present paper presents a complete bidding strategy for a PSP unit combining the benefits of price arbitrage with the participation in the ancillary services market. Compared to [5], the present paper has the following original contributions: (a) a medium-term model (inspired by [6]) is applied, combining the seasonality of market prices with water value concepts, to find the optimal level of water in the reservoir at the end of each week; (b) forecasting techniques were applied to predict natural water inflows, day-ahead and frequency restoration reserve; (c) does not assume prior knowledge of the amount of mobilized FRR capacity; (d) describes an evaluation module that replicates the "real" operation of the power plant and enables an accurate evaluation of the profit.

The paper is organized as follows: section II presents the 
overall architecture for the bidding strategies, describing the medium and short-term models and detailing the evaluation phase; section III details the case study and results; section IV enumerates the obtained conclusions.

\section{Bidding Strategies Framework}

The overall bidding strategy framework for the participation of a PSP unit in the day-ahead and frequency restoration reserve (FRR) markets is depicted in Figure 1. This strategy is divided into three modules:

\section{Medium-term Optimization}

Allocation of the water from natural inflows considering a one year time horizon. It uses weekly forecasts for the average market price and natural water inflows. The outcome from the model is the optimal weekly reservoir level for the entire year.

\section{Short-term Optimization}

Combining information provided by forecasts of natural water inflows and market prices of the day-ahead and FRR markets with technical information of the pump-hydro unit, this model defines the operational set points for the PSP, aiming to maximize its revenue. The model uses the output of the medium-term model to constraint the level of the reservoir at the end of the optimization period.

\section{Evaluation Module}

Using real price and energy data from the electricity market, this module calculates the revenue of the system. The reservoir level is updated Using the information of the realized water inflows and energy requested for FRR.

Considering an iterative approach for the strategy implementation, the updated level of the reservoir obtained from the evaluation module is used as the initial state in the mediumterm model and all the process is repeated for the next day.

\section{A. Input/Output Curves}

Figure 2 illustrates this I/O curves for turbine mode, using a methodology adapted from [7] and [5]. The same process is made to find the pump mode I/O curves. Taking the generation curve as example, the technical boundaries are defined by the maximum and minimum power generation $\left(\bar{g}^{d}, g^{d}\right)$ and water flow $\left(\bar{q}^{d}, q^{d}\right)$. Using these values it is possible to compute the slope of the curve, i.e. energy coefficient $\delta^{d}$, which multiplied by the water flow translates into power generation. The same process is used to define the energy coefficient for pumping $\left(\delta^{p}\right)$.

This is an approximation of the real I/O curves and it is only possible to do it by assuming that the PSP operates with small head variation. Otherwise the non-linear relation between water flow and head would increase the complexity of the problem and it would not be possible to solve this problem using Mixed Integer Linear Programming.

\section{B. Optimization Models}

1) Medium-term Optimization: The operation of a PSP unit relies in the correct management of its only resource, i.e. water.

The medium-term optimization model was inspired in the work of [6] and takes into account the seasonality and variability of the natural water inflows combined with day-ahead market prices forecasts to find the optimal allocation of the water resource for one year, considering weekly periods (i.e., 52 weeks).

The objective function (1) aims at maximize the revenue of the system, which results from the multiplication of the hydro unit generation power $g_{w}^{d}$ by the day-ahead market price $\pi_{D A_{w}}$ for a period of 52 weeks $(w=52)$. Given the weekly nature of the optimization periods, all the variables present in this model are represented by their weekly average value, with the exception of the natural water inflows, which are represented by their weekly sum.

$$
\max \text { revenue }=\max \sum_{w=1}^{w=52}\left[g_{w}^{d} \cdot \pi_{D A_{w}}\right]
$$

The amount of water present in the reservoir for each week $Q_{w}$ is updated using (2), where $q_{w}^{d}$ is the average water discharge flow from the hydro turbine and $a_{w}$ sum of the weekly natural water inflows in the reservoir.

$$
Q_{w}=Q_{w-1}-q_{w}^{d}+a_{w}
$$

The initial and final level of the reservoir (3)(4) must be set, being $Q^{\text {start }}$ the realized volume in the reservoir at the time of the optimization and $Q^{\text {end }}$ a predefined value, in this paper the medium level of the reservoir is used.

$$
\begin{gathered}
Q_{0}=Q^{\text {start }} \\
Q_{52}=Q^{\text {end }}=\frac{Q+\bar{Q}}{2}
\end{gathered}
$$

Equations (5)-(7) are the constraints responsible to impose the technical limits of the hydroelectric power $\left(g^{d}, g^{d}\right)$, water discharge flow $\left(q^{d}, \bar{q}^{d}\right)$ and reservoir level $(Q, \bar{Q})$.

$$
\begin{aligned}
& \underline{g}^{d} \leq g_{w} \leq \bar{g}^{d} \\
& \underline{q}^{d} \leq q_{w} \leq \bar{q}^{d} \\
& Q \leq Q_{w} \leq \bar{Q}
\end{aligned}
$$

A seasonal bootstrapping technique, following the work from [8], is used to simulate the yearly time series for day-ahead market prices and natural water inflows, since traditional forecasting methods, such as neural networks or ARIMA, would result in large errors due to the extent of the forecast horizon. The seasonal bootstrap method involves nonparametric randomly resampling historical data, by taking segments of the historical time series and pasting them together to form a new artificial series. Thus preserving any seasonal or trend patterns as well as the inherent serial correlation. 


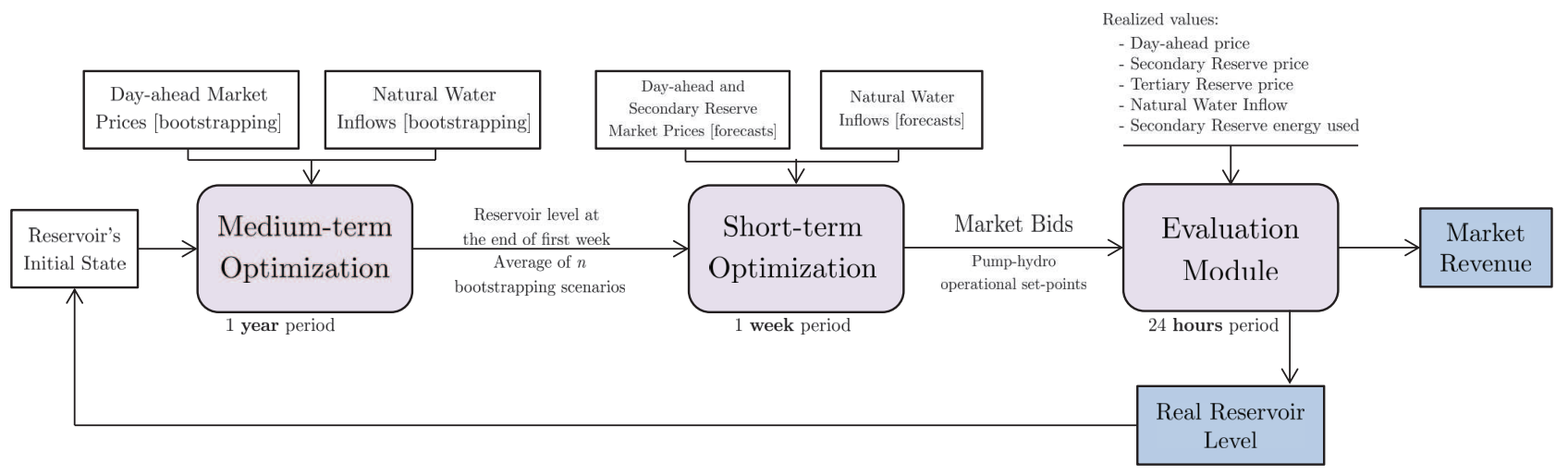

Figure 1. Architecture of the Optimization Framework

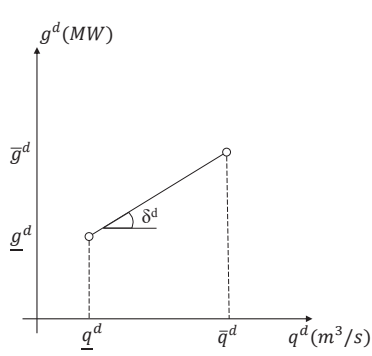

Figure 2. Input/Output curve of variable speed PSP - inspired in [5]

To increase the robustness of the bootstrapping technique, a large set of scenarios (i.e. $n=500$ ) are created and all of them are used as input in the medium-term optimization algorithm. The end result is the average of all scenarios and the reservoir level obtained for the end of the first week is then used as constraint in the short-term optimization model to define the reservoir level at the end of the optimization period.

2) Short-term Optimization: The formulation of the shortterm optimization model presumes that it is not possible to determine a priori the amount of electrical energy used from the available reserve band. Thus, the revenue for the FRR is represented in the objective function only for the reserve capacity price. Following the same logic, the amount of reserve band made available to perform FRR does not affect the rate of discharge/pumping water flow or the amount of water available in the reservoir. To avoid situations where the energy used in the frequency restoration would result in the violation of a reservoir level it is assumed that a portion of the reservoir is allocated solely for the purpose of frequency restoration.

The objective function of the Short-term Optimization Model (8) aims to maximize the revenue of the system for a period of one week ( $t=168$ hours). There are two different sources of income: the day-ahead market (DA) and the FRR market. The revenue of the first term results from the difference of the energy generated $\left(g_{D A}^{d}\right)$ and the electrical energy consumed by the pump unit $\left(g_{D A}^{p}\right)$ multiplied by the DA market price $\left(\pi_{D A}\right)$. In the objective function it is only considered the remuneration of the regulation band of the FRR reserve. In other words, it is only considered the availability of the FRR regulation band. Thus, the revenue results from the multiplication of the available power for upward $\left(u s^{d}, u s^{p}\right)$ and downward $\left(d s^{d}, d s^{p}\right)$ regulation by the FRR market price $\left(\pi_{S E C}\right)$

$$
\begin{aligned}
\max \sum_{t=1}^{t=168}[ & \left(g_{D A, t}^{d}-g_{D A, t}^{p}\right) \cdot \pi_{D A, t} \\
& \left.\quad+\left(u s_{t}^{d}+u s_{t}^{p}+d s_{t}^{d}+d s_{t}^{p}\right) \cdot \pi_{S E C, t}\right]
\end{aligned}
$$

Equations (9)-(12) define the amount of water flow discharged $\left(q^{d}\right)$ from the upper reservoir or pumped $\left(q^{p}\right)$ into the upper reservoir by the unit. Binary variables $\left(o n^{d}, o n^{p}\right)$ indicate when the unit is operating as turbine or pump and constraint (13) ensures that simultaneous operation does not occur.

$$
\begin{gathered}
q a_{t}^{d} \leq o n_{t}^{d} \cdot\left(\bar{q}^{d}-\underline{q}^{d}\right) \\
q a_{t}^{p} \leq o n_{t}^{p} \cdot\left(\bar{q}^{p}-\underline{q}^{p}\right) \\
q_{t}^{d}=q a_{t}^{d}+o n_{t}^{d} \cdot \underline{q}^{d} \\
q_{t}^{p}=q a_{t}^{p}+o n_{t}^{p} \cdot \underline{q}^{p} \\
u_{t}^{d}+u_{t}^{p} \leq 1
\end{gathered}
$$

The relation between water flow and power is characterized by Equations (14) and (15), which results from the multiplication of the energy coefficient $\left(\delta^{d}, \delta^{p}\right)$ by the water flow, as explained in Section II-A.

$$
\begin{aligned}
& g_{D A, t}^{d}=o n_{t}^{d} \cdot \underline{g}^{d}+q a_{D A, t}^{d} \cdot \delta^{d} \\
& g_{D A, t}^{p}=o n_{t}^{p} \cdot \underline{g}^{p}+q a_{D A, t}^{p} \cdot \delta^{p}
\end{aligned}
$$


With the known values of discharge and pumping flow for each optimization period, it is possible to update the reservoir level using Equation (16). The starting level is defined by the real amount of water in the reservoir and the value for the final value results from the medium-term optimization model, as explained in Section II-B1.

$$
\begin{gathered}
Q_{t}=Q_{t-1}+\left(q_{t}^{p}-q_{t}^{d}\right)+a_{t} \\
Q_{0}=Q^{\text {start }} \\
Q_{168}=Q^{\text {end }}
\end{gathered}
$$

Equations (19)-(22) ensure that the power available for FRR regulation band added to the day-ahead bid does not violate the maximum or minimum limits for the pump-hydro unit. The day-ahead operation itself must be within the technical boundaries of the unit, since the regulation band may not be activated (or used). This is guaranteed by Equations (23) and (24).

$$
\begin{gathered}
g_{D A, t}^{d}+u s_{t}^{d} \leq o n_{t}^{d} \cdot \bar{g}^{d} \\
g_{D A, t}^{d}-d s_{t}^{d} \geq o n_{t}^{d} \cdot \underline{g}^{d} \\
g_{D A, t}^{p}+d s_{t}^{p} \leq o n_{t}^{p} \cdot \bar{g}^{p} \\
g_{D A, t}^{p}-u s_{t}^{p} \geq o n_{t}^{p} \cdot \underline{g}^{p} \\
o n_{t}^{d} \cdot \underline{g}^{d} \leq g_{D A, t}^{d} \leq o n_{t}^{d} \cdot \bar{g}^{d} \\
o n_{t}^{d} \cdot \underline{g}^{p} \leq g_{D A, t}^{p} \leq o n_{t}^{p} \cdot \bar{g}^{p}
\end{gathered}
$$

The ancillary services market in the Iberian peninsula are separately operated by the transmission system operator (TSO) of each country. In Portugal, the TSO forces that the amount for upward secondary reserve must account for $2 / 3$ of the total band, leaving $1 / 3$ for downward regulation. This rule is imposed by constraint (25).

$$
\left(u s_{t}^{d}+u s_{t}^{p}\right)=2 \cdot\left(d s_{t}^{d}+d s_{t}^{p}\right)
$$

In order to forecast the day-ahead energy and FRR prices, as well as the natural water inflows, a forecasting model based on the component-wise gradient boosting (GB) algorithm [9] was used. It conducts numerical optimization, via steepest-descent, in function space by using a user-defined base learner recurrently on modified data that is the output from the previous iterations. Following the optimization phase, the final solution is a linear combination of the base learners. The main feature of this algorithm is that the base learner selects only one predictor among all in each iteration and can includes different types of base learners, such as linear effects or smooth effects (e.g., P-splines). Therefore, this algorithm is capable of modeling non-linear relations and conducts feature selection. To train and apply this model the component-wise GB from R package "mboost" [10] was used.
3) Evaluation Module: To evaluate the performance of the bidding strategy an evaluation module, where the markets bids determined by the optimization problem are applied to real market conditions, is implemented. Observed prices from the electricity market are used to evaluate the economic performance and observed natural water inflows used to update reservoir level. Despite the weekly period of the short-term optimization model, the MIBEL only accepts bids for the dayahead, so in this evaluation module only the first 24 hours bids are considered.

The revenue is calculate according to Equation (26), where the first term accounts the revenue from the day-ahead market and the second the revenue from the FRR regulation band. The last two terms accommodate the revenue from the amount of energy used from the available FRR reserve. This value is estimated based on the ratio of the energy used and the total amount of FRR reserve contracted (27)(28).

$$
\begin{aligned}
\text { revenue }=\sum_{t=1}^{t=24}[ & \left(g_{D A, t}^{d}-g_{D A, t}^{p}\right) \cdot \pi_{D A, t} \\
& +\left(u s_{t}^{d}+u s_{t}^{p}+d s_{t}^{d}+d s_{t}^{p}\right) \cdot \pi_{S E C, t} \\
& +\% \uparrow \cdot\left(u s_{t}^{d}+u s_{t}^{p}\right) \cdot \pi_{T E R \_u p, t} \\
& \left.-\% \downarrow \cdot\left(d s_{t}^{d}+d s_{t}^{p}\right) \cdot \pi_{T E R \_d o w n, t}\right]
\end{aligned}
$$

$$
\begin{gathered}
\% \uparrow_{t}=\frac{\text { upward FRR energy used }(t)}{\text { upward FRR band contracted }(t)}(\%) \\
\downarrow_{t}=\frac{\text { downward FRR energy used }(t)}{\text { downward FRR band contracted }(t)}(\%)
\end{gathered}
$$

Together with the realized values of the natural water inflows $\left(a^{\text {real }}\right)$, the amount of electrical energy used is also relevant to update the level of the reservoir, since, for instance, the use of the PSP unit to perform downward regulation results in more water stored in the reservoir (29).

$$
\begin{gathered}
\hat{Q}_{t}-Q_{t}^{r e a l}=\sum_{t=1}^{t=24}\left[\left(\hat{a}-a^{r e a l}\right)-\% \uparrow_{t} \cdot\left(\frac{u s_{t}^{d}}{\delta^{d}}+\frac{u s_{t}^{p}}{\delta^{p}}\right)\right. \\
\left.+\% \downarrow_{t} \cdot\left(\frac{d s_{t}^{d}}{\delta^{d}}+\frac{d s_{t}^{p}}{\delta^{p}}\right)\right]
\end{gathered}
$$

\section{CASE STUdy}

The optimization models described in section II-B were applied to a real PSP unit located in the north of Portugal, with technical characteristics presented in Table I.

Real data for the MIBEL market prices provided by $\mathrm{REN}^{1}$ and $\mathrm{REE}^{2}$ and natural water inflows were used to evaluate the performance of the bidding strategies. The period between May 2014 and May 2015 was considered.

\footnotetext{
${ }^{1}$ www.mercado.ren.pt

${ }^{2}$ www.esios.ree.es
} 


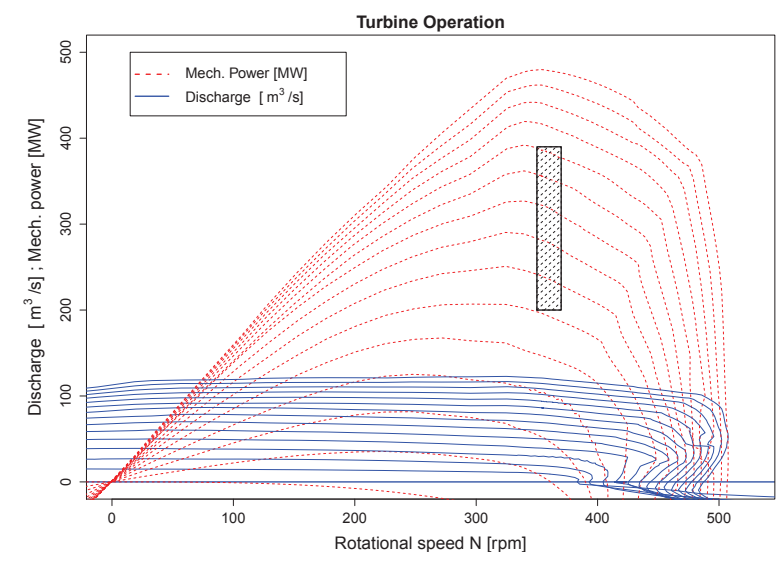

(a)

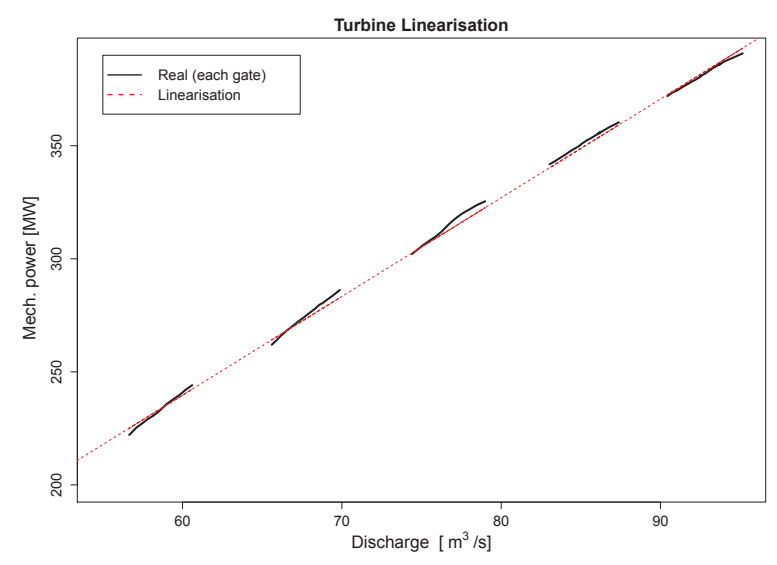

(b)

Figure 3. Turbine mode: (a) Normalized s-shape curve (for max efficiency) (b) Linearization

TABLE I. TECHNICAL CHARACTERISTICS OF THE PSP UNIT

\begin{tabular}{lccc}
\hline \hline & $\max$ & $\min$ & \\
\hline Turbine & 390 & 200 & {$[\mathrm{MW}]$} \\
Pump & 390 & 300 & {$[\mathrm{MW}]$} \\
Reservoir & 92.1 & 76.3 & {$\left[\mathrm{hm}^{3}\right]$} \\
\hline \hline
\end{tabular}

To obtain the energy coefficients, like explained in Section II-A, we used s-shape characteristic curves, provided by the manufacture of the machine, which gives the relation between water flow and power for all the gate openings.

When operating in pump-mode the machine does not change the gate opening, thus we use solely the curve for the gate opening with higher efficiency to find $\delta^{p}$. However, in turbinemode the gate is changed regularly, so, using the s-shape curves depicted in Figure $3 \mathrm{a}$ and restricting the operation to the machine technical limits (highlighted by the shaded rectangle), we can get the relation of water discharge and power generation for each gate, like depicted in Figure $3 \mathrm{~b}$. The slope of the linearisation (dotted red line) is the energy coefficient in generation mode, $\delta^{d}$.

In Figure 4 it is possible to observe the outcome of typical optimization day. The dotted lines correspond to the day-ahead market bids and the shaded area to the FRR reserve band, both obtained by the short-term optimization. Bold lines represent the actual power generated or consumed by the pump-hydro unit, being the difference between the DA bid and the actual power the amount of energy used to perform FRR control.

The revenue obtained by the system was compared with a PSP unit with fixed speed and the results are described in Table II and Figure 5.

As expected, with variable speed PSP the revenue from the day-ahead market suffers a small decrease, but it is largely compensated by the revenue from the FRR market. Adding the revenue of the two markets, a PSP with variable speed more than duplicates the revenue in comparison with a fixed speed PSP.

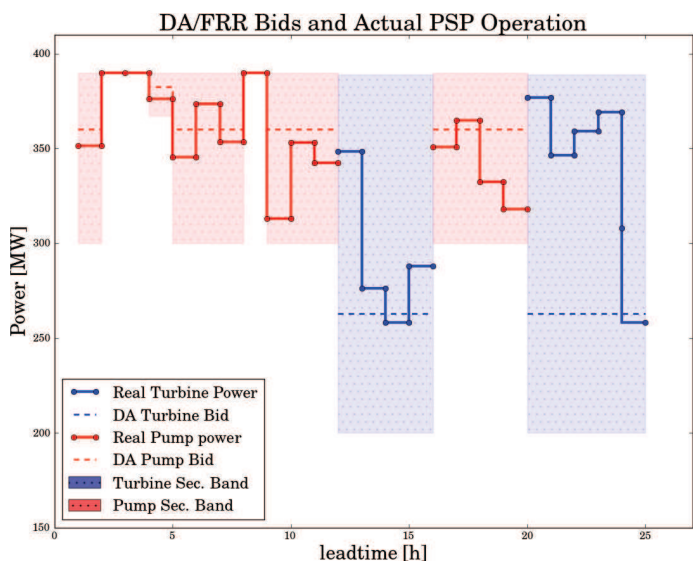

Figure 4. DA and FRR bids and actual PSP operation points

TABLE II. SYSTEM REVENUE FROM DA AND FRR, CONSIDERING

\begin{tabular}{lccc}
\multicolumn{4}{c}{ VARIABLE AND FIXED SPEED } \\
\hline \hline & Variable Speed & Fixed Speed \\
\hline Day-ahead & 45.18 & 48.9 & EUR $10^{6}$ \\
Frequency R. R. & 44.33 & - & EUR $10^{6}$ \\
Total & 94.08 & 48.9 & EUR $10^{6}$ \\
\hline \hline
\end{tabular}

Since the amount of energy used from the reserve band was not considered in the short-term optimization model, it is possible that during the operating day the reservoir level would be above or below its technical limits. Figure 6 shows a histogram of the difference between the amount of water expected after the optimization process and the actual level after the operation. This deviation indicates that if we limit the maximum reservoir level to a value $1 \mathrm{hm}^{3}$ below the maximum and $1 \mathrm{hm}^{3}$ above the minimum, it is expected that the technical constraints would not be violated. 


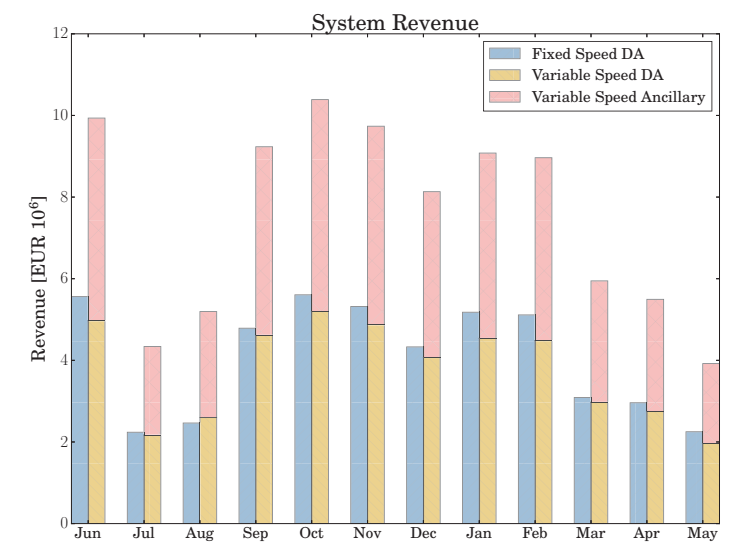

Figure 5. System Revenue from DA and FRR, considering variable and fixed speed

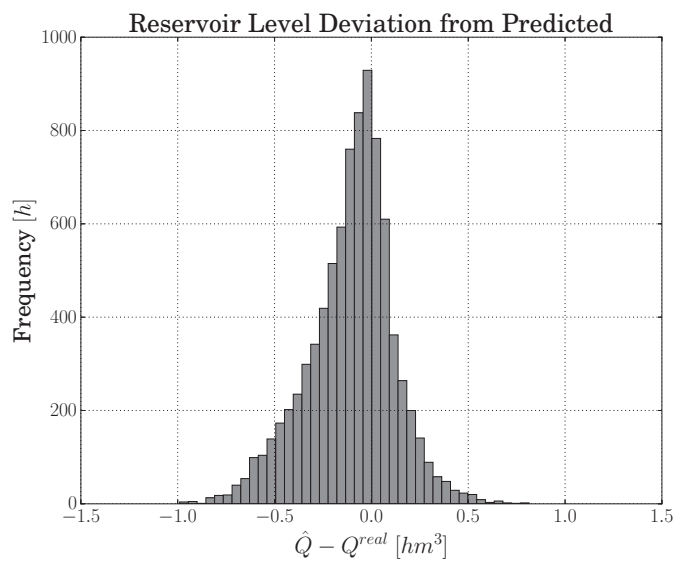

Figure 6. Reservoir level deviation from the predicted

\section{CONCLuSion}

This paper presents a complete optimization strategy for the optimization of variable speed PSP on the day-ahead and FRR markets. The strategy combines a medium-term model that allocates the water resource throughout the year with a short-term model that defines the operating point and FRR band of the PSP unit in order to maximize the power plant revenue.

Considering an evaluation period of one year, the economic results show that the participation of these units in FRR could double their revenue. It was also concluded that, even disregarding the impact in the reservoir level of the energy used for FRR, the actual amount of water never surpassed the $1 \mathrm{hm}^{3}$ from the level expected from the optimization and the observed one. The obtained results show that the participation of these unis in ancillary markets increases by $92 \%$ the system revenue and diversify their bidding strategies. This work did not considered hydraulic or electrical losses, and assumed that bids in the electricity market were always accepted, leaving room for improvement in the assessment of real energy used from the regulation band in the evaluation module.

Future work consists in including replacement reserve bids in the optimization model and combine PSP with renewable energy power plants in order to present combined bids in the ancillary services market (e.g., virtual power plant approach).

\section{ACKNOWLEDGMENTS}

The authors graciously thank the partners from the FP7 European Project HYPERBOLE for the valuable comments about the pumped storage power modeling and EDP Produção for the provision of technical data and natural water inflow time series. A special mention to Christophe Nicolet from Power Vision Engineering for the contribution in modeling the I/O curves and Jiri Koutnik from Voith for providing the s-shape curve.

\section{REFERENCES}

[1] N. Sivakumar, D. Das, and N. Padhy, "Variable speed operation of reversible pump-turbines at kadamparai pumped storage plant - a case study," Energy Conversion and Management, vol. 78, pp. 96-104, Feb. 2014.

[2] A. Beguin, C. Nicolet, B. Kawkabani, and F. Avellan, "Virtual power plant with pumped storage power plant for renewable energy integration," in Proc. of the XXI International Conference on Electrical Machines (ICEM 2014), Berlin, Germany, Sept. 2014.

[3] C. Nicolet, A. Beguin, B. Kawkabani, Y. Pannatier, A. Schwery, and F. Avellan, "Variable speed and ternary units to mitigate wind and solar intermittent production," in Proc. of the 2014 HydroVision International, Nashville, TN, Jul. 2014.

[4] J. I. Pérez-Díaz, M. Chazarra, J. García-González, G. Cavazzini, and A. Stoppato, "Trends and challenges in the operation of pumpedstorage hydropower plants," Renewable and Sustainable Energy Reviews, vol. 44, pp. 767-784, Apr. 2015.

[5] M. Chazarra, J. I. Perez-Diaz, and J. Garcia-Gonzalez, "Optimal operation of variable speed pumped storage hydropower plants participating in secondary regulation reserve markets," in Proc. of the 11th International Conference on the European Energy Market (EEM14), Krakow, Poland, May 2014.

[6] J. C. Sousa, V. T. Mendes, and J. T. Saraiva, "Estimation of the remuneration of hydro plants in a market environment using an iterative under-relaxation approach," in Proc. of the IEEE PowerTech Conference 09, Bucharest, Romania, Jun. 2009.

[7] J. García-González, E. Parrilla, A. Mateo, and R. Moraga, "Building optimal generation bids of a hydro chain in the day-ahead electricity market under price uncertainty," in Proc, of the 9th International Conference on Probabilistic Methods Applied to Power Systems (PMAPS 2006), Stockholm, Sweden, Jun. 2006.

[8] R. Hyndman and S. Fan, "Density forecasting for long-term peak electricity demand," IEEE Transactions on Power Systems, vol. 25, no. 2, pp. 1142-1153, 2010 .

[9] P. Bhlmann, "Boosting for high-dimensional linear models," Ann. of Stat., vol. 34, no. 2, pp. 559-583, 2006.

[10] B. Hofner, A. Mayr, N. Robinzonov, and M. Schmid, "Model-based boosting in R: A hands-on tutorial using the R package mboost," Computational Statistics, vol. 29, pp. 3-35, 2014. 